Original Research Paper

\title{
Reliability Equivalence Factors of a Parallel System Subject to Weibull Distribution Using Multivariate FGM Copula
}

\author{
Neama Salah Youssef Temraz \\ Department of Mathematics, Faculty of Science, Tanta University, Tanta, Egypt
}

Article history

Received: 07-07-2017

Revised: $14-10-2017$

Accepted: 31-01-2018

Email: sci_neama55@yahoo.com

\begin{abstract}
This paper analyze a parallel system consisted of $\mathrm{n}$ dependent components with lifetimes follow Weibull distribution. FGM Copula in multivariate case is used to generate the reliability function of the original system. Reduction method is introduced to improve system reliability. Other methods of hot, cold and warm duplication are established to improve system reliability. An application using real time data is introduced to show the results and compare between different improvement methods.
\end{abstract}

Keywords: FGM Copula, Reliability, Parallel System, Dependent Units, Probability Density Function, Distribution Function, Hazard Rate Function, Weibull Distribution, Cold Standby, Warm Standby, Hot Standby, Reduction Method, Equivalence Factors

\section{Introduction}

In systems analysis, reliability can be increased by using components with high reliability or increase the number of redundant components. There are many techniques that used in increasing efficiency of a system such as reduction and redundancy. In reduction method, system reliability can be increased by reducing the failure rates of some of its units by a factor $\rho$ such that $\rho$ is a number between zero and one (i.e., $0<\rho<1$ ) (Sarhan et al., 2004; Sarhan, 2009). Redundancy method is classified to hot, warm and cold standby duplication methods.

In hot duplication method, some of the system devices are duplicated in parallel. In cold duplication method, some of the system devices are duplicated by a cold standby component via a switch which can be perfect or imperfect. In warm duplication method, some of the system devices are connected by a warm standby component via a switch which can be perfect or imperfect (Sarhan, 2009).

Råde (1989) introduced the concepts of reliability equivalence factors. Råde (1993a; 1993b) applied this concept for parallel and series systems with independent and identical components with lifetimes follow exponential distribution. Reliability equivalence factor is defined as the factor by which the failure rates of some of system units should be reduced in order to reach equality of the reliability of another better system (Sarhan, 2005).

Sarhan (2000) obtained the reliability equivalence factors of a series system consists of $n$ independent and non-identical units by using the reliability function and mean time to failure as characteristics to compare different system designs. Sarhan (2002) extended the concept of reliability equivalence factors from simple series and parallel systems to some complex systems where he considered a radar system in an aircraft which consists of three independent and non-identical components with constant failure rates. Sarhan (2004) introduced the reliability equivalence factors of a bridge network system. Sarhan and Mustafa (2006) proposed the reliability equivalence factors of a series system which consists of $\mathrm{n}$ independent and non-identical units. Sarhan et al. (2008) introduced reliability equivalence factors of a parallel-series system assuming that the failure rates of the system units are constant. Sarhan (2009) introduced reliability equivalence factors of a general series-parallel system with independent units have lifetimes follow exponential distribution.

Xia and Zhang (2007) studied reliability equivalence factors of parallel system assuming that the failure rates of the system units are dependent of time with lifetimes follow gamma distribution. El-Damcese (2009) introduced the reliability equivalence factors of a seriesparallel system when the system units are independent and identical with lifetimes follow Weibull distribution. Reliability equivalence factors for some systems with mixture Weibull failure rates were introduced by Mustafa (2009). 
Khan and Jan (2015) introduced reliability evaluation of an engineering system using modified Weibull distribution. Mustafa and El-Faheem (2014) presented reliability equivalence factors of a system with mixture of $\mathrm{n}$ independent and non-identical lifetimes with delay time. Reliability equivalence factor of a parallel system subject to time varying failure rates is studied by El-Damcese and Alltifi (2013). Ezzati and Rasouli (2015) improved system reliability using linear-exponential distribution function. El-Damcese and Ayoub (2011) obtained reliability equivalence factors for $\mathrm{n}$ independent and identical units of a parallel system by using bivariate Weibull model. Reliability equivalence factors for a general series-parallel system in Burr type $X$ distribution are derived by Migdadi and Al-Batah (2014). Shawky et al. (2013) introduced reliability equivalence factors in case of exponentiated exponential distribution.

In this paper, a study of a parallel system consisting of $n$ dependent and non-identical components is introduced. Reliability function of the original system is derived by using the concepts of copula subject to Weibull distribution. Reliability function of the original system is improved according to reduction, hot duplication, warm and cold duplication methods. Reliability equivalence factors are introduced to compare between different system designs. Numerical illustration and real time data application is discussed to show the results obtained in this paper.

\section{Copula Definitions}

\section{Definition (1): Copula (Mangey and Singh, 2009)}

A d-dimensional copula is a distribution function on $[0,1]^{d}$ with standard uniform marginal distributions. Let $C(u)=C\left(u_{1}, u_{2}, \ldots, u_{d}\right)$ be the distribution functions which are copulas. Hence $C$ is a mapping of the form $C:[0,1]^{d}$ $\rightarrow[0,1]$, i.e. a mapping of the unit hypercube into the unit interval. The following three properties must hold:

- $\quad C\left(u_{1}, u_{2}, \ldots, u_{d}\right)$ is increasing in each component $u_{i}$

- $\quad C\left(1, \ldots, 1, u_{i}, 1, \ldots, 1\right)=u_{i}$ for all $i \in\{1, \ldots, d\}, u_{i} \in[0,1]$

- For all $\left(a_{1}, \ldots, a_{d}\right),\left(b_{1}, \ldots b_{d}\right) \in[0,1]$ with $a_{i} \leq b_{i}$ we have:

$$
\sum_{i_{1}=1}^{2} \ldots \sum_{i_{d}=1}^{2}(-1)^{i_{1}+\ldots i_{d}} C\left(u_{i_{1}}, \ldots, u_{i_{d}}\right) \geq 0
$$

where, $u_{i_{1}}=a_{i}, u_{i_{2}}=b_{i}$ for all $\mathrm{i} \in\{1, \ldots, d\}$.

\section{Theorem 1: Sklar (Mangey and Singh, 2009)}

Let $F$ be a joint distribution function with margins $F_{1}, \ldots, F_{d}$ (not necessarily continuous). Then there exists a copula $C:[0,1]^{d} \rightarrow[0,1]$, such that for all $x_{1}, \ldots x_{d}$ in $\overline{\mathfrak{R}}=[-\infty, \infty]$ :

$F\left(x_{1}, \ldots, x_{d}\right)=C\left(F_{1}\left(x_{1}\right), \ldots, F_{d}\left(x_{d}\right)\right)$

If the margins are continuous then $C$ is unique; otherwise $C$ is uniquely determined on $\operatorname{Ran} F_{1} \times \ldots \times$ Ran $F_{d}$, where $\operatorname{Ran} F_{i}$ denotes the range of $F_{i}: \operatorname{Ran} F_{i}=F_{i}(\overline{\mathfrak{R}})$. Conversely, if $C$ is a copula and $F_{1}, \ldots, F_{d}$ are distribution functions, then the function $F$ defined in (1) is a joint distribution function with margins $F_{1}, \ldots, F_{d}$.

\section{Definition (2): (Mangey and Singh, 2009)}

If $\mathrm{F}$ is a joint distribution function with marginals $F_{1}, \ldots, F_{d}$ and theorem (Sklar) holds, we say that $\mathrm{C}$ is a copula of $\mathrm{F}$ (or a random vector $X \sim F$ ). If the marginals are continuous then $C$ is the unique copula of $F$ (or $X$ ). The copula is the distribution function of the component wise probability transformed random vector. Alternatively, we can evaluate (1) at the arguments $x_{i}=$ $F_{i}\left(u_{i}\right), 0 \leq u_{i} \leq 1, i=1, \ldots, d$ and use the property of the generalized inverse to obtain:

$C\left(u_{1}, \ldots, u_{d}\right)=F\left(F_{1}\left(u_{1}\right), \ldots, F_{d}\left(u_{d}\right)\right)$

where, $F^{\leftarrow}$ is the generalized inverse of $F$.

Definition (3): Bivariate Farlie-Gumbel-Morgenstern Coupla

Farlie-Gumbel-Morgenstern copula (Nelsen, 2006) is defined by:

$C\left(u_{1}, u_{2}\right)=u_{1} u_{2}\left[1+\phi\left(1-u_{1}\right)\left(1-u_{2}\right)\right]$

where, $(-1 \leq \phi \leq 1)$ and the independence corresponds $\phi=0$.

\section{Definition (4): Multivariate Farlie-Gumbel- Morgenstern Coupla}

Gauvreau and Pagano (1997) considered a d-variate Farlie-Gumbel-Morgenstern (FGM) copula. The multivariate FGM copula is:

$C\left(u_{1}, u_{2}, \ldots, u_{d} ; \vartheta\right)=\left[1+\sum_{k=2}^{d} \sum_{1 \leq j_{1}<\ldots<j_{k} \leq d} \vartheta_{j_{1}, \ldots, j_{k}}\left(1-u_{j_{1}}\right) \ldots\left(1-u_{j_{k}}\right)\right] \prod_{k=1}^{d} u_{k}$

where, $\left(-1 \leq \vartheta_{j_{1}, \ldots, j_{k}} \leq 1\right)$ for all $j_{1}, \ldots, j_{k}$.

\section{Original System}

A system consisted of $n$ dependent and nonidentical units connected in parallel which considered as shown in Fig. 1. 


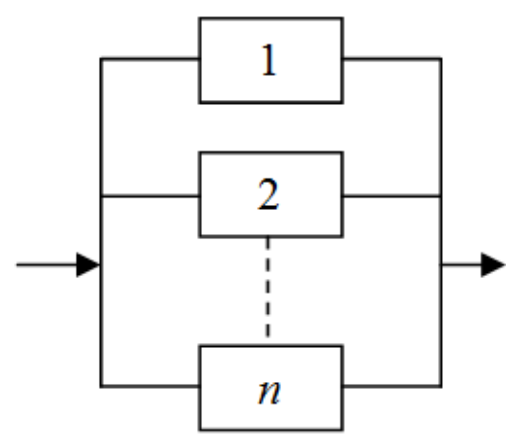

Fig. 1: Parallel system

Assume that the life time of each unit is $T=\left(T_{1}\right.$, $\left.T_{2}, \ldots, T_{n}\right)$ and $F_{i}(t)$ is the distribution function of $T_{i}$. The joint distribution function is $\operatorname{Pr}\left\{T_{1} \leq t_{1}, T_{2} \leq t_{2}, \ldots, T_{n} \leq\right.$ $\left.t_{n}\right\}$ and the reliability function of each component is $R_{i}(t), i=1,2, \ldots, n$. From the Sklar theorem, there is an $\mathrm{n}-$ dimensional copula function $C$ that makes $\operatorname{Pr}\left\{T_{1} \leq t_{1}, T_{2}\right.$ $\left.\leq t_{2}, \ldots, T_{n} \leq t_{n}\right\}=C^{n}\left(F_{1}\left(t_{1}\right), F_{2}\left(t_{2}\right), \ldots, F_{n}\left(t_{n}\right)\right)$, when each unit of a system is in parallel, the life time of the system is the largest in all the units, that is $T=\max \left(T_{1}, T_{2}, \ldots, T_{n}\right)$, then the reliability of a parallel system can be expressed as (Hai et al., 2016):

$$
\begin{aligned}
& R(t)=\operatorname{Pr}\left\{\max \left(T_{1}, T_{2}, \ldots, T_{n}\right)>t\right\}=1-\operatorname{pr}\left\{\max \left(T_{1}, T_{2}, \ldots, T_{n}\right) \leq t\right\} \\
& =1-C^{n}\left(F_{1}(t), F_{2}(t), \ldots, F_{n}(t)\right)=1-C^{n}\left(1-R_{1}(t), 1-R_{2}(t), \ldots, 1-R_{n}(t)\right)
\end{aligned}
$$

According the previous definition and multivariate FGM copula family, the reliability function of the parallel system of $\mathrm{n}$ dependent units is deduced as follows:

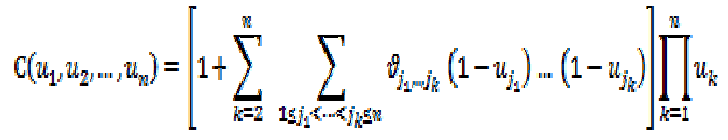

where, $\left(-1 \leq \vartheta_{j_{1}, \ldots j_{k}} \leq 1\right)$ for all $j_{1}, \ldots, j_{k}$.

It can be written as follows:

$c\left(u_{1}, u_{2}, \ldots, u_{n} ; \vartheta\right)=$
$\left[\begin{array}{l}1+\sum_{1 \leq j_{1}<j_{2} \leq n}^{n} \vartheta_{j_{1}, j_{2}}\left(1-u_{j_{1}}\right)\left(1-u_{j_{2}}\right) \\ +\sum_{\substack{1 \leq j_{1}<j_{2}<j_{3} \leq n \\+\ldots+\vartheta_{1, \ldots, n}}} \vartheta_{j_{1}, j_{2}, j_{3}}\left(1-u_{1}\right) \ldots\left(1-u_{j_{1}}\right)\left(1-u_{j_{2}}\right)\left(1-u_{j_{3}}\right)\end{array}\right] \prod_{k=1}^{n} u_{k}$

Density function of multivariate FGM copula is deduced in the following steps:

$c\left(u_{1}, u_{2}, \ldots, u_{n} ; \vartheta\right)=\frac{\partial^{n} C\left(u_{1}, u_{2}, \ldots, u_{n} ; \vartheta\right)}{\partial u_{1} \partial u_{2} \ldots \partial u_{n}}$ and hence:

$$
\begin{aligned}
& c\left(u_{1}, u_{2}, \ldots, u_{n} ; \vartheta\right)= \\
& 1+\sum_{1 \leq j_{1}<j_{2} \leq n}^{n} \vartheta_{j_{1}, j_{2}}\left(1-2 u_{j_{1}}\right)\left(1-2 u_{j_{2}}\right) \\
& \sum_{1 \leq j_{1}<j_{2}<j_{3} \leq n}^{n} \vartheta_{j_{1}, j_{2}, j_{3}}\left(1-2 u_{j_{1}}\right)\left(1-2 u_{j_{2}}\right)\left(1-2 u_{j_{3}}\right) \\
& +\ldots+\vartheta_{1, \ldots, n}\left(1-2 u_{1}\right) \ldots\left(1-2 u_{n}\right)
\end{aligned}
$$

The reliability function of the system is defined as:

$$
R(t)=1-C\left(u_{1}, u_{2}, \ldots, u_{n} ; \vartheta\right)
$$

where, $u_{i}=F_{i}(t)$ is the distribution function of $T_{i}, i=$ $1,2, \ldots, n$. If the life time of each unit follows Weibull distribution with two parameters, then the distribution function and the reliability function of each unit will be given by:

$$
\begin{aligned}
& u_{j_{k}}=1-e^{-\left(t / \lambda_{j_{k}}\right)^{\beta} j_{j_{k}}}, 1-u_{j_{k}}=e^{-\left(t / \lambda_{j_{k}}\right)^{\beta} j_{j_{k}}}, \\
& \text { for } k=1,2, \ldots, n, \lambda_{j_{k}}, \beta_{j_{k}}>0, t \geq 0
\end{aligned}
$$

where, $\lambda_{j_{k}}$ and $\beta_{j_{k}}$ are the scale and shape parameters, respectively. The reliability function of the parallel system is obtained as follows:

$$
\begin{aligned}
& R(t)=1-\left[1+\sum_{1 \leq j_{1}<j_{2} \leq n}^{n} \vartheta_{j_{1}, j_{2}} e^{-\left[\left(t / \lambda_{j_{1}}\right)^{\beta} j_{1}+\left(t / \lambda_{j_{2}}\right)^{\beta / j_{2}}\right]}\right. \\
& \left.+\sum_{1 \leq j_{1}<j_{2}<j_{3} \leq n}^{n} \vartheta_{j_{1}, j_{2}, j_{3}} e^{-\left[\left(t / \lambda_{j_{1}}\right)^{\beta} j_{1}+\left(t / \lambda_{j_{2}}\right)^{\beta} j_{2}+\left(t / \lambda_{j_{3}}\right)^{\beta / 3}\right]}\right] \\
& \left.+\ldots+\vartheta_{1, \ldots, n} e^{-\sum_{k=1}^{n}\left(t / \lambda_{k}\right)^{\beta_{k}}}\right] \\
& \prod_{k=1}^{n}\left[1-e^{-\left(t / \lambda_{k}\right)^{\beta_{k}}}\right]
\end{aligned}
$$

The reliability function of the parallel system is plotted for the following data:

$$
\begin{aligned}
& n=3, \vartheta_{12}=0.6, \vartheta_{13}=0.5, \vartheta_{23}=0.7, \vartheta_{123}=1, \lambda_{2}=0.06, \\
& \lambda_{3}=0.08, \beta_{1}=0.4, \beta_{2}=0.5, \beta_{3}=0.7,0 \leq \lambda_{1} \leq 0.1,0 \leq t \leq 5
\end{aligned}
$$

and the result is shown in Fig. 2. 


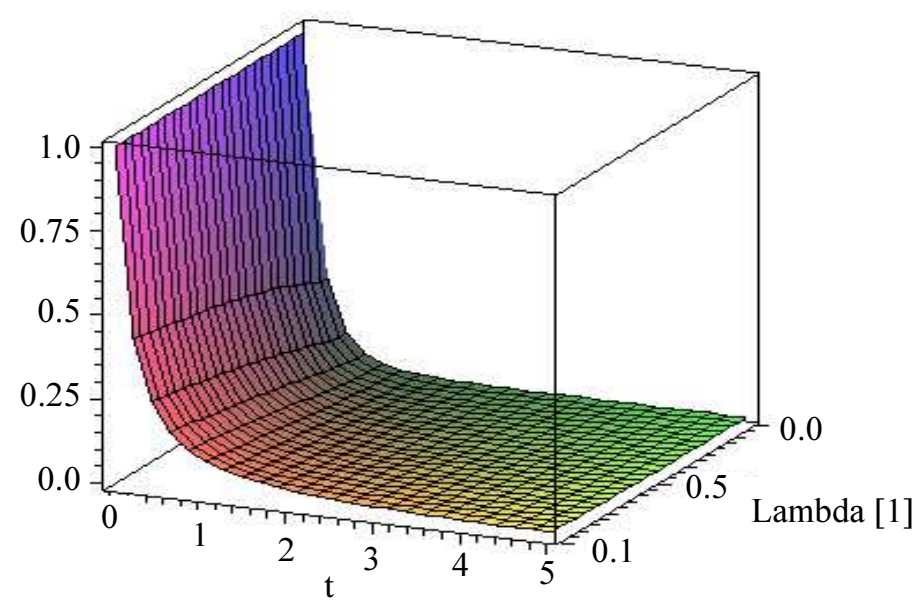

Fig. 2: Reliability function versus time and the failure rate $\lambda_{1}$

The density function of multivariate FGM copula is deduced as follows:

$$
\begin{aligned}
& c\left(u_{1}, u_{2}, \ldots, u_{n} ; \vartheta\right)=1+\sum_{1 \leq j_{1}<j_{2} \leq n}^{n} \vartheta_{j_{1}, j_{2}}\left(2 e-\left(t / \lambda_{j_{1}}\right)^{\beta}-1\right)\left(2 e-\left(t / \lambda_{j_{2}}\right)^{\beta / j_{2}}-1\right) \\
& +\sum_{1 \leq j_{1}<j_{2}<j_{3} \leq n}^{n} \vartheta_{j_{1}, j_{2}, j_{3}}\left(2 e^{-\left(t / \lambda_{j_{1}}\right)^{\beta} \beta_{1}}-1\right)\left(2 e^{-\left(t / \lambda_{j_{2}}\right)^{\beta / j_{2}}}-1\right) \\
& \left(2 e^{\left(-\left(t / \lambda_{j_{3}}\right)^{\beta} / \beta_{3}\right.}-1\right)+\ldots+\vartheta_{1, \ldots, n} \prod_{k=1}^{n}\left(2 e^{-\left(t / \lambda_{k}\right)^{\beta_{k}}}-1\right)
\end{aligned}
$$

The probability density function of the parallel system will be given from the following relation (Liu and Fan, 2016):

$$
f(t)=c\left(F_{1}(t), F_{2}(t), \ldots, F_{n}(t)\right) \prod_{i=1}^{n} f_{i}(t)
$$

where, $f_{i}(t)$ is the marginal probability density function and $c\left(F_{1}(t), F_{2}(t), \ldots, F_{n}(t)\right)$ is the joint PDF of the copula function. Then PDF function of the original system is obtained as follows:

$$
\begin{aligned}
& f(t)=\left[\begin{array}{l}
1+\sum_{1 \leq j_{1}<j_{2} \leq n}^{n} \vartheta_{j_{1}, j_{2}}\left(2 e^{-\left(t / \lambda_{j_{1}}\right)^{\beta} j_{1}}-1\right)\left(2 e^{-\left(t / \lambda_{j_{2}}\right)^{\beta / j_{2}}}-1\right) \\
+\sum_{1 \leq j_{1}<j_{2}<j_{3} \leq n}^{n} \vartheta_{j_{1}, j_{2}, j_{3}}\left(2 e^{-\left(t / \lambda_{j_{1}}\right)^{\beta} j_{1}}-1\right)\left(2 e^{-\left(t / \lambda_{j_{2}}\right)^{\beta} j_{2}}-1\right) \\
\left(2 e^{-\left(t / \lambda_{j_{3}}\right)^{\beta / \beta_{3}}}-1\right)+\cdots+\vartheta_{1, \ldots, n} \prod_{k=1}^{n}\left(2 e^{-\left(t / \lambda_{k}\right)^{\beta_{k}}}-1\right)
\end{array}\right] \\
& \prod_{k=1}^{n}\left[\frac{\left.\beta_{k}\left(\frac{t}{\lambda_{k}}\right)^{\beta_{k}-1} e^{-\left(t / \lambda_{k}\right)^{\beta_{k}}}\right]}{\lambda_{k}}\right]
\end{aligned}
$$

Using the same data in reliability function, results for the density function of the parallel system can be obtained which is shown in Fig. 3.

The hazard rate function of the original system can be deduced as follows:

$$
h(t)=\frac{f(t)}{R(t)}
$$

and hence:

$$
\begin{aligned}
& h(t)=\left\{\left[1+\sum_{1 \leq j_{1}<j_{2} \leq n}^{n} \vartheta_{j_{1}, j_{2}}\left(2 e^{-\left(t / \lambda_{j_{1}}\right)^{\beta} j_{1}}-1\right)\left(2 e^{-\left(t / \lambda_{j_{2}}\right)^{\beta} j_{2}}-1\right)\right.\right. \\
& +\sum_{1 \leq j_{1}<j_{2}<j_{3} \leq n}^{n} \vartheta_{j_{1}, j_{2}, j_{3}}\left(2 e^{-\left(t / \lambda_{j_{1}}\right)^{\beta} j_{1}}-1\right)\left(2 e^{-\left(t / \lambda_{j_{2}}\right)^{\beta} j_{2}}-1\right) \\
& \left.\left(2 e^{\left.-\left(t / \lambda_{j_{3}}\right)^{\beta}\right)_{3 / 3}}-1\right)+\ldots+\vartheta_{1, \ldots, n} \prod_{k=1}^{n}\left(2 e^{-\left(t / \lambda_{k}\right)^{\beta_{k}}}-1\right)\right] \\
& \left.\prod_{k=1}^{n}\left[\frac{\beta_{k}}{\lambda_{k}}\left(\frac{t}{\lambda_{k}}\right)^{\beta_{k}-1} e^{-\left(t / \lambda_{k}\right)^{\beta_{k}}}\right]\right\} \div \\
& \left\{1-\left[1+\sum_{1 \leq j_{1}<j_{2} \leq n}^{n} \vartheta_{j_{1}, j_{2}} e^{-\left[\left(t / \lambda_{j_{1}}\right)^{\beta} j_{1}\left(t / \lambda_{j_{2}}\right)^{\beta} j_{2}\right.}\right]\right. \\
& \left.+\sum_{1 \leq j_{1}<j_{2}<j_{3} \leq n}^{n} \vartheta_{j_{1}, j_{2}, j_{3}} e^{\left[\left(t / \lambda_{j_{1}}\right)^{\beta}+\left(t / \lambda_{j_{2}}\right)^{\beta}\right)^{\beta}+\left(t / \lambda_{j_{s}}\right)^{\beta} j_{s}}\right] \\
& \left.\left.+\ldots+\vartheta_{1, \ldots, n} e^{-\sum_{k=1}^{n}\left(t / \lambda_{k}\right)^{\beta_{k}}}\right] \prod_{k=1}^{n}\left[1-e^{-\left(t / \lambda_{k}\right)^{\beta_{k}}}\right]\right\}
\end{aligned}
$$




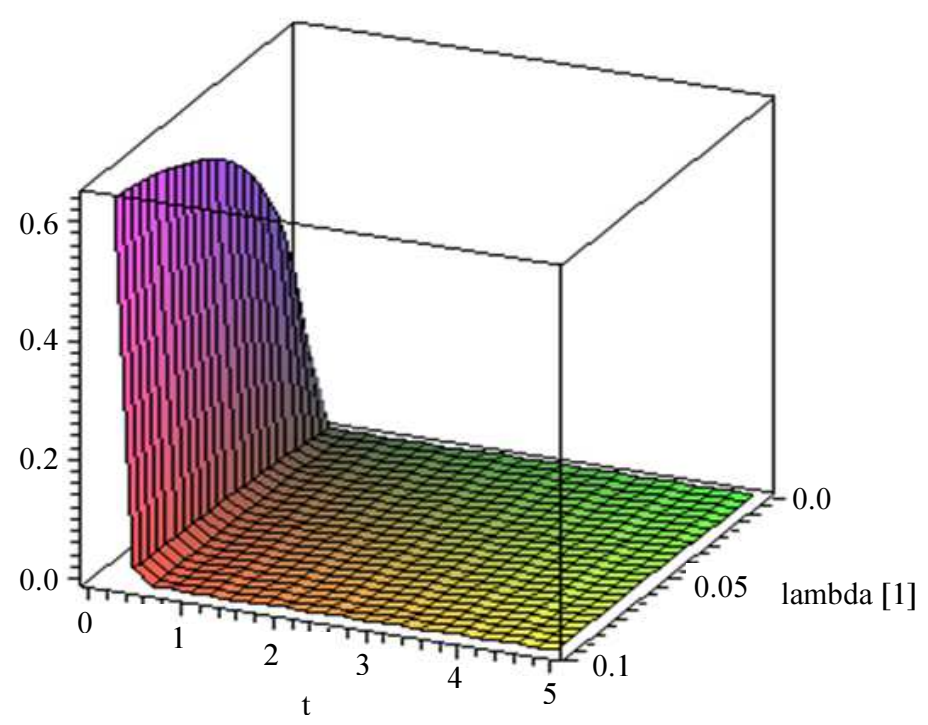

Fig. 3: Probability density function $f(t)$ versus time and the failure rate $\lambda_{1}$
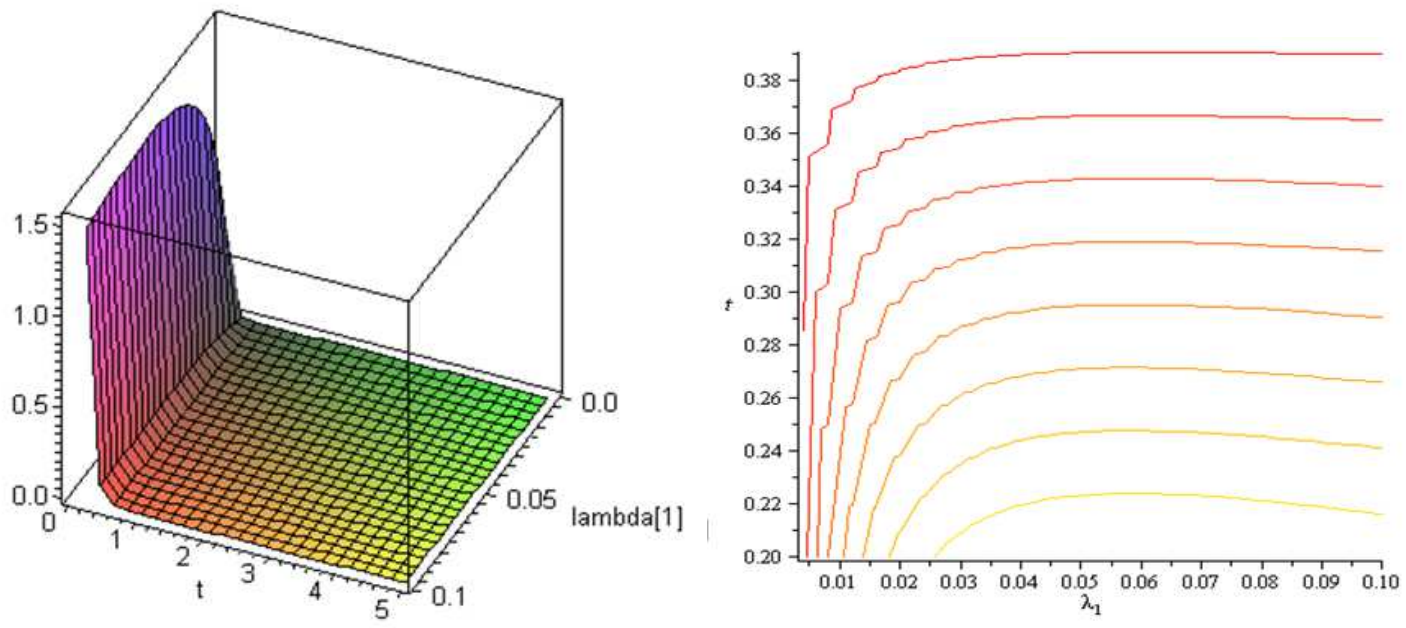

Fig. 4: Hazard rate function $h(t)$ versus time and the failure rate $\lambda_{1}$

Using the same data in reliability function, the results for the hazard rate function of the parallel system can be obtained which is shown in Fig. 4.

\section{Reduction Method}

In this method, it is supposed that the failure rates of a set $r$ of the units of the system are decreased by multiplying by a factor $\rho, 0<\rho<1$ and hence system reliability function is obtained as follows:

$R_{\text {red. }}(t)=1-\left[1+\sum_{1 \leq j_{1}<j_{2} \leq n}^{n} \vartheta_{j_{1}, j_{2}} e^{-\left[\delta_{j_{1}}\left(t / \lambda_{j_{1}}\right)^{\beta} j_{1}+\delta_{j_{2}}\left(t / \lambda_{j_{2}}\right)^{\beta} j_{2}\right.}\right]$

$$
\begin{aligned}
& +\sum_{1 \leq j_{1}<j_{2}<j_{3} \leq n}^{n} \vartheta_{j_{1}, j_{2}, j_{3}} e^{-\left[\delta_{j_{1}}\left(t / \lambda_{j_{1}}\right)^{\beta} j_{1_{1}}+\delta_{j_{2}}\left(t / \lambda_{j_{2}}\right)^{\beta} j_{j_{2}}+\delta_{j_{3}}\left(t / \lambda_{j_{3}}\right)^{\beta_{3}}\right]}+\ldots . \\
& \left.+\vartheta_{1, \ldots, n} e^{-\left[\sum_{k \in r} \rho_{k}\left(t / \lambda_{k}\right)^{\beta_{k}}+\sum_{k \varepsilon r}\left(t / \lambda_{k}\right)^{\beta_{k}}\right]}\right] \\
& \prod_{k \in r}\left[1-e^{-\rho_{k}\left(t / \lambda_{k}\right)^{\beta_{k}}}\right] \prod_{k \notin r}\left[1-e^{-\rho_{k}\left(t / \lambda_{k}\right)^{\beta_{k}}}\right]
\end{aligned}
$$

where:

$$
\delta_{j_{k}}=\left\{\begin{array}{ll}
\rho_{j_{k}}, & \text { if } j_{k} \in r \\
1, & \text { if } j_{k} \notin r
\end{array}, \text { for } k=1,2, \ldots, n\right.
$$




\section{Cold Standby Duplication Method}

In this method, it is supposed that a set $c$ of components of the system are duplicated with a cold standby unit via a perfect switch. The reliability function of the improved system is obtained as follows:

$$
\begin{aligned}
& R_{\text {cold }}(t)=1-\left[1+\sum_{1 \leq j_{1}<j_{2} \leq n}^{n} \vartheta_{j_{1}, j_{2}} R_{j_{1}}(t) R_{j_{2}}(t)+\right. \\
& \sum_{1 \leq j_{1}<j_{2}<j_{3} \leq n}^{n} \vartheta_{j_{1}, j_{2}, j_{3}} R_{j_{1}}(t) R_{j_{2}}(t) R_{j_{3}}(t)+\ldots \\
& \left.+\vartheta_{1, \ldots, n} \prod_{k \in c}\left(1+\left(\frac{t}{\lambda_{k}}\right)^{\beta_{k}}\right) e^{-\left[\sum_{k=1}^{n}\left(t / \lambda_{k}\right)^{\beta_{k}}\right]}\right] \\
& \prod_{k \in c}\left[1-\left(1+\left(\frac{t}{\lambda_{k}}\right)^{\beta_{k}}\right) e^{-\left(t / \lambda_{k}\right)^{\beta_{k}}} \prod_{k \notin c} 1-e^{-\left(t / \lambda_{k}\right)^{\beta_{k}}}\right]
\end{aligned}
$$

where:

$$
R_{j_{k}}(t)=\left\{\begin{array}{cl}
\left(1+\left(\frac{t}{\lambda_{j_{k}}}\right)^{\beta_{j_{k}}}\right) e^{-\left(t / \lambda_{j_{k}}\right) \beta_{j_{k}}}, & \text { if } j_{k} \in c \\
e^{-\left(t / \lambda_{j_{k}}\right)^{\beta} j_{k}}, & \text { if } j_{k} \notin c
\end{array}\right.
$$

\section{Warm Standby Duplication Method}

In this method, it is supposed that a set $w$ of components of the system are duplicated with a warm standby unit via a perfect switch. The reliability function of the improved system is obtained as follows:

$$
\begin{aligned}
& R_{\text {warm }}(t)=1-\left[1+\sum_{1 \leq j_{1}<j_{2} \leq n}^{n} \vartheta_{j_{1}, j_{2}} R_{j_{1}}(t) R_{j_{2}}(t)+\right. \\
& \sum_{1 \leq j_{1}<j_{2}<j_{3} \leq n}^{n} \vartheta_{j_{1}, j_{2}, j_{3}} R_{j_{1}}(t) R_{j_{2}}(t) R_{j_{3}}(t)+\ldots+\vartheta_{l_{1, \ldots, n}} \\
& \prod_{k \in w}\left(e^{\left\{\left(t / \lambda_{k}\right)^{\beta_{k}}+\left(t / \lambda_{s_{k}}\right)^{\beta} s_{k}\right\}}+e^{\left(t / \lambda_{k}\right)^{\beta_{k}}}\left\{1-e^{-\left(t / \lambda_{s_{k}}\right)^{\beta} s_{k}}\right.\right.
\end{aligned}
$$

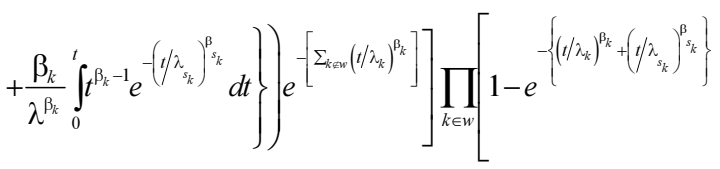

$$
\begin{aligned}
& \left.-e^{-\left(t / \lambda_{k}\right)^{\beta_{k}}}\left\{1-e^{-\left(t / \lambda_{s_{k}}\right)^{\beta} s_{k}}+\frac{\beta}{\lambda^{\beta}} \int_{0}^{t} t^{\beta-1} e^{\left.-\left(t / \lambda_{s_{k}}\right)^{\beta}\right)_{k}} d t\right\}\right]\left[1-e^{-\left(t / \lambda_{k}\right)^{\beta_{k}}}\right]
\end{aligned}
$$

where:

$\lambda_{s_{k}}, \beta_{s_{k}}$ are the scale and shape parameters of the $k$-th warm standby unit. and:

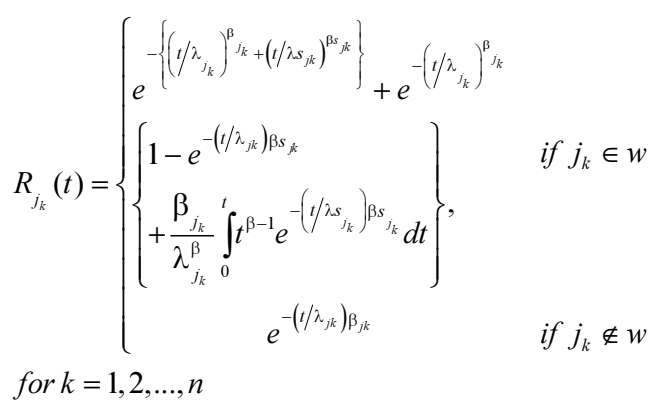

\section{Hot Standby Duplication Method}

In this method, it is supposed that a set of $h$ components of the system are duplicated with a hot standby unit. The reliability function of the improved system is obtained as follows:

$$
\begin{aligned}
& R_{h o t}(t)=1-\left[\begin{array}{l}
1+\sum_{1 \leq j_{1}<j_{2} \leq n}^{n} \vartheta_{j_{1}, j_{2}} R_{j_{1}}(t) R_{j_{2}}(t)+ \\
\sum_{1 \leq j_{1}<j_{2}<j_{3} \leq n}^{n} \vartheta_{j_{1}, j_{2}, j_{3}} R_{j_{1}}(t) R_{j_{2}}(t) R_{j_{3}}(t)+\ldots
\end{array}\right. \\
& \left.+\vartheta_{1, \ldots, n} \prod_{k \in h}\left(2-e^{-\left(t / \lambda_{k}\right)^{\beta_{k}}}\right) e^{-\left[\sum_{k \in h}\left(t / \lambda_{k}\right)^{\beta_{k}}\right]}\right] \\
& \prod_{k \in h}\left[1-\left(2-e^{-\left(t / \lambda_{k}\right)^{\beta_{k}}}\right) e^{-\left(t / \lambda_{k}\right)^{\beta_{k}}}\right] \\
& \prod_{k \notin h}\left[1-e^{-\left(t / \lambda_{k}\right)^{\beta_{k}}}\right]
\end{aligned}
$$

where:

$$
R_{j_{k}}(t)=\left\{\begin{array}{cc}
\left(2-e^{-\left(t / \lambda_{k}\right)^{\beta_{k}}}\right) e^{-\left(t / \lambda_{k}\right)^{\beta_{k}}}, & \text { if } j_{k} \in h \\
e^{\left.-\left(t / \lambda_{j_{k}}\right)^{\beta}\right)_{k}}, & \text { if } j_{k} \notin h
\end{array}\right.
$$

\section{Reliability Equivalence Factors}

Suppose that at given time $T=t$ the reliability function of the improved system according the reduction method can be written using the expansion of the exponential function as the following formula:

$$
\begin{aligned}
& R_{\text {red. }}(t)=1-\left[1+\sum_{1 \leq j_{1}<j_{2} \leq n}^{n} \vartheta_{j_{1}, j_{2}}\right. \\
& \sum_{m=0}^{\infty} \frac{(-1)^{m}\left[\delta_{j_{1}}\left(t / \lambda_{j_{1}}\right)^{\beta / 4}+\delta_{j_{2}}\left(t / \lambda_{j_{2}}\right)^{\beta_{1 / 2}}\right]^{m}}{m !}
\end{aligned}
$$




$$
\begin{aligned}
& \begin{array}{r}
(-1)^{m}\left[\begin{array}{l}
\delta_{j_{1}}\left(t / \lambda_{j_{1}}\right)^{\beta_{j_{1}}} \\
+\delta_{j_{2}}\left(t / \lambda_{j_{2}}\right)^{\beta_{j_{2}}} \\
+\delta_{j_{3}}\left(t / \lambda_{j_{3}}\right)^{\beta_{3}}
\end{array}\right]^{m} \\
m !
\end{array} \\
& \left.+\vartheta_{1, \ldots, n} \sum_{m=0}^{\infty} \frac{(-1)^{m}\left[\sum_{k \in r} \rho_{k}\left(t / \lambda_{k}\right)^{\beta_{k}}\right]^{m}}{m !} e^{-\left[\sum_{k \varepsilon r}\left(t / \lambda_{k}\right)^{\beta_{k}}\right]}\right] \\
& \prod_{k \in r}\left[1-\sum_{m=0}^{\infty} \frac{(-1)^{m}\left[\rho_{k}\left(t / \lambda_{k}\right)^{\beta_{k}}\right]^{m}}{m !}\right] \prod_{k \notin r}\left[1-e^{-\left(t / \lambda_{k}\right)^{\beta_{k}}}\right]=\varphi(\rho)
\end{aligned}
$$

where:

$$
\delta_{j_{k}}=\left\{\begin{array}{cl}
\rho_{j_{k}}, & \text { if } j_{k} \in r \\
1 & \text { if } j_{k} \notin r,
\end{array} \text { for } k=1,2, \ldots, n\right.
$$

Suppose that at given time $T=t$, the reliability function of improved systems according cold, warm and hot duplication methods are given by $R_{\text {cold }}(t)=\alpha$, $R_{\text {warm }}(t)=\gamma, R_{h o t}(t)=\sigma$, respectively. The reliability equivalence factors can be obtained as the solutions of the following nonlinear equations:

$$
\varphi\left(\rho_{\text {cold }}\right)=\alpha, \varphi\left(\rho_{\text {warm }}\right)=\gamma, \varphi\left(\rho_{\text {hot }}\right)=\sigma
$$

Since these equations are non-linear with respect to $\rho$ and is cannot be solved analytically hence numerical solutions can be obtained for the equivalence factors $\rho$.

\section{Application}

The introduced model will be discussed and results for the reliability function in different cases will be obtained assuming that the system consists of three components $(n=3)$. The reliability function of the original model will be given by:

$$
\begin{aligned}
& R(t)=1-\left\{1+\vartheta_{12} e^{-\left[\left(t / \lambda_{1}\right)^{\beta_{1}}+\left(t / \lambda_{2}\right)^{\beta_{2}}\right]}+\vartheta_{13} e^{-\left[\left(t / \lambda_{1}\right)^{\beta_{1}}+\left(t / \lambda_{3}\right)^{\beta_{3}}\right]}\right. \\
& \left.+\vartheta_{23} e^{-\left[\left(t / \lambda_{2}\right)^{\beta_{2}}+\left(t / \lambda_{3}\right)^{\beta_{3}}\right]}+\vartheta_{123} e^{-\left[\left(t / \lambda_{1}\right)^{\beta_{1}}+\left(t / \lambda_{2}\right)^{\beta_{2}}+\left(t / \lambda_{3}\right)^{\beta_{3}}\right]}\right\} \\
& \left(1-e^{-\left(t / \lambda_{1}\right)^{\beta_{1}}}\right)\left(1-e^{-\left(t / \lambda_{2}\right)^{\beta_{2}}}\right)\left(1-e^{-\left(t / \lambda_{3}\right)^{\beta_{3}}}\right)
\end{aligned}
$$

The reliability function of the improved system according the reduction method assuming that $r=\{1,2\}$ will be given by:

$$
\begin{aligned}
& R_{\text {red. }}(t)=1-\left\{1+\vartheta_{12} e^{-\left[\rho_{1}\left(t / \lambda_{1}\right)^{\beta_{1}}+\rho_{2}\left(t / \lambda_{2}\right)^{\beta_{2}}\right]}\right. \\
& +\vartheta_{13} e^{-\left[\rho_{1}\left(t / \lambda_{1}\right)^{\beta_{1}}+\left(t / \lambda_{3}\right)^{\beta_{3}}\right]} \\
& +\vartheta_{23} e^{-\left[\rho_{2}\left(t / \lambda_{2}\right)^{\beta_{2}}+\left(t / \lambda_{3}\right)^{\beta_{3}}\right]} \\
& \left.+\vartheta_{123} e^{-\left[\rho_{1}\left(t / \lambda_{1}\right)^{\beta_{1}}+\rho_{2}\left(t / \lambda_{2}\right)^{\beta_{2}}+\left(t / \lambda_{3}\right)^{\beta_{3}}\right]}\right\} \\
& \left(1-e^{-\rho_{1}\left(t / \lambda_{1}\right)^{\beta_{1}}}\right)\left(1-e^{-\rho_{2}\left(t / \lambda_{2}\right)^{\beta_{2}}}\right)\left(1-e^{-\left(t / \lambda_{3}\right)^{\beta_{3}}}\right)
\end{aligned}
$$

where, the reduction method is applied on the first and second units only.

The reliability function of the improved system according the cold standby duplication method assuming that $c=\{1,2\}$ will be given by:

$$
\begin{aligned}
& R_{\text {cold. }}(t)=1-\left\{\begin{array}{l}
1+\vartheta_{12}\left(1+\left(\frac{t}{\lambda_{1}}\right)^{\beta_{1}}\right) e^{-\left[\left(t / \lambda_{1}\right)^{\beta_{1}}+\left(t / \lambda_{2}\right)^{\beta_{2}}\right]} \\
\left(1+\left(\frac{t}{\lambda_{2}}\right)^{\beta_{2}}\right)
\end{array}\right. \\
& +\vartheta_{13}\left(1+\left(\frac{t}{\lambda_{1}}\right)^{\beta_{1}}\right) e^{-\left[\left(t / \lambda_{1}\right)^{\beta_{1}}+\left(t / \lambda_{3}\right)^{\beta_{3}}\right]} \\
& +\vartheta_{23}\left(1+\left(\frac{t}{\lambda_{2}}\right)^{\beta_{2}}\right) e^{-\left[\left(t / \lambda_{2}\right)^{\beta_{2}}+\left(t / \lambda_{3}\right)^{\beta_{3}}\right]} \\
& +\vartheta_{123}\left(1+\left(\frac{t}{\lambda_{1}}\right)^{\beta_{1}}\right) \\
& \left(1+\left(\frac{t}{\lambda_{2}}\right)^{\beta_{2}}\right) \\
& \left.e^{-\left[\left(t / \lambda_{1}\right)^{\beta_{1}}+\left(t / \lambda_{2}\right)^{\beta_{2}}\left(t / \lambda_{3}\right)^{\beta_{3}}\right]}\right] \\
& \left(1-\left(1+\left(\frac{t}{\lambda_{1}}\right)^{\beta_{1}}\right) e^{-\left(t / \lambda_{1}\right) \beta_{1}}\right) \\
& \left(1-\left(1+\left(\frac{t}{\lambda_{2}}\right)^{\beta_{2}}\right) e^{-\left(t / \lambda_{2}\right)^{\beta_{2}}}\right)\left(1-e^{-\left(t / \lambda_{3}\right)^{\beta_{3}}}\right)
\end{aligned}
$$

The reliability function of the improved system according the warm standby duplication method assuming that $w=\{1,2\}$ will be given by: 


$$
\begin{aligned}
& R_{\text {warm }}(t)=1-\left\{1+\vartheta_{12}\left(e^{-\left\{\left(t / \lambda_{1}\right)^{\beta_{1}}+\left(t / \lambda_{s_{1}}\right)^{\beta_{s}}\right.}\right\}+e^{-\left(t / \lambda_{1}\right)^{\beta_{1}}}\left\{1-e^{-\left(t / \lambda_{s_{1}}\right)^{\beta_{s_{1}}}}+\frac{\beta_{1}}{\lambda^{\beta_{1}}} \int_{0}^{t} t^{\beta_{1}-1} e^{-\left(t / \lambda_{s_{1}}\right)^{\beta_{s_{1}}}} d t\right\}\right) \\
& \left.\left(e^{-\left\{\left(t / \lambda_{2}\right)^{\beta_{2}}+\left(t / \lambda_{s_{2}}\right)^{\beta} s_{2}\right.}\right\}+e^{-\left(t / \lambda_{2}\right)^{\beta_{2}}}\left\{1-e^{-\left(t / \lambda_{s_{2}}\right)^{\beta} s_{2}}+\frac{\beta_{2}}{\lambda^{\beta_{2}}} \int_{0}^{t} t^{\beta_{2}-1} e^{-\left(t / \lambda_{s_{2}}\right)^{\beta} s_{2}} d t\right\}\right) \\
& +\vartheta_{13}\left(e^{-\left\{\left(t / \lambda_{1}\right)^{\beta_{1}}+\left(t / \lambda_{s_{1}}\right)^{\beta_{s_{1}}}\right\}}+e^{-\left(t / \lambda_{1}\right)^{\beta_{1}}}\left\{1-e^{-\left(t / \lambda_{s_{1}}\right)^{\beta_{s}}}+\frac{\beta_{1}}{\lambda^{\beta_{1}}} \int_{0}^{t} t^{\beta_{1}-1} e^{-\left(t / \lambda_{s_{1}}\right) \beta_{s_{1}}} d t\right\} e^{-\left(t / \lambda_{s}\right) \beta_{s}}\right. \\
& +\vartheta_{23}\left(e^{-\left\{\left(t / \lambda_{2}\right)^{\beta_{2}}+\left(t / \lambda_{s_{2}}\right)^{\beta_{2}}\right\}}+e^{-\left(t / \lambda_{2}\right)^{\beta_{2}}}\left\{1-e^{-\left(t / \lambda_{s_{2}}\right)^{\beta_{s_{2}}}}+\frac{\beta_{2}}{\lambda^{\beta_{2}}} \int_{0}^{t} t^{\beta_{2}-1} e^{-\left(t / \lambda_{s_{2}}\right)^{\beta_{s_{2}}}} d t\right\} e^{-\left(t / \lambda_{s}\right)^{\beta_{s}}}\right. \\
& +\vartheta_{123}\left(e^{-\left\{\left(t / \lambda_{1}\right)^{\beta_{1}}+\left(t / \lambda_{s_{1}}\right)^{\beta_{s_{1}}}\right\}}+e^{-\left(t / \lambda_{2}\right)^{\beta_{1}}}\left\{1-e^{-\left(t / \lambda_{s_{1}}\right)^{\beta_{1}}}+\frac{\beta_{1}}{\lambda^{\beta_{1}}} \int_{0}^{t} t^{\beta_{1}-1} e^{-\left(t / \lambda_{s_{1}}\right)^{\beta_{s}}} d t\right\}\right) \\
& \left(e^{-\left\{\left(t / \lambda_{2}\right)^{\beta_{2}}+\left(t / \lambda_{s_{2}}\right)^{\beta_{s_{2}}}\right\}}+e^{-\left(t / \lambda_{2}\right)^{\beta_{2}}}\left\{1-e^{-\left(t / \lambda_{s_{2}}\right)^{\beta_{2}}}+\frac{\beta_{2}}{\lambda^{\beta_{2}}} \int_{0}^{t} t^{\beta_{2}-1} e^{-\left(t / \lambda_{s_{2}}\right)^{\beta} s_{2}} d t\right\} e^{-\left(t / \lambda_{2}\right)^{\beta_{s}}}\right\} \\
& \left.\left(1-\left(e^{-\left\{\left(t / \lambda_{1}\right)^{\beta_{1}}+\left(t / \lambda_{s_{1}}\right)^{\beta} s_{1}\right.}\right\}+e^{-\left(t / \lambda_{1}\right)^{\beta_{1}}}\left\{1-e^{-\left(t / \lambda_{s_{1}}\right)^{\beta_{s_{1}}}}+\frac{\beta_{4}}{\lambda^{\beta_{2}}} \int_{0}^{t} t^{\beta_{1}-1} e^{-\left(t / \lambda_{s_{1}}\right)^{\beta} s_{1}} d t\right\}\right)\right)\left(1-\left(e^{\left\{-\left(t / \lambda_{2}\right)^{\beta_{2}}+\left(t / s_{2}\right)^{\beta_{s_{2}}}\right.}\right\}\right.
\end{aligned}
$$

The reliability function of the improved system according the hot standby duplication method assuming that $h=\{1,2\}$ will be given by:

$$
\begin{aligned}
& R_{h o t}(t)=1-\left\{1+\vartheta_{12}\left(2-e^{-\left(t / \lambda_{1}\right)^{\beta_{1}}}\right)\right. \\
& \left(2-e^{-\left(t / \lambda_{2}\right)^{\beta_{2}}}\right) e^{-\left[\left(t / \lambda_{1}\right)^{\beta_{1}}+\left(t / \lambda_{2}\right)^{\beta_{2}}\right]} \\
& \left.+\vartheta_{13}\left(2-e^{-\left(t / \lambda_{1}\right)^{\beta_{1}}}\right) e^{-\left[\left(t / \lambda_{1}\right)^{\beta_{1}}+\left(t / \lambda_{3}\right)^{\beta_{3}}\right.}\right] \\
& +\vartheta_{23}\left(2-e^{-\left(t / \lambda_{2}\right)^{\beta_{2}}}\right) e^{-\left[\left(t / \lambda_{2}\right)^{\beta_{2}}+\left(t / \lambda_{3}\right)^{\beta_{3}}\right]} \\
& +\vartheta_{123}\left(2-e^{-\left(t / \lambda_{1}\right)^{\beta_{1}}}\right)\left(2-e^{-\left(t / \lambda_{2}\right)^{\beta_{2}}}\right) \\
& \left.\left.e-^{\left[\left(t / \lambda_{1}\right)^{\beta_{1}}+\left(t / \lambda_{2}\right)^{\beta_{2}}+\left(t / \lambda_{3}\right)^{\beta_{3}}\right]}\right\}\right\}\left(1-\left(2-e^{-\left(t / \lambda_{1}\right)^{\beta_{1}}}\right) e-^{\left(t / \lambda_{1}\right)^{\beta_{1}}}\right) \\
& \left(1-\left(2-e^{-\left(t / \lambda_{2}\right)^{\beta_{2}}}\right) e^{-\left(t / \lambda_{2}\right)^{\beta_{2}}}\right)\left(1-e^{-\left(t / \lambda_{3}\right)^{\beta_{3}}}\right)
\end{aligned}
$$

\section{Reliability Equivalence Factors}

Approximated reliability equivalence factors will be given from the following relation:

$$
\begin{aligned}
& R_{\text {red. }}(t) \cong 1-\left[1+\vartheta_{12} \sum_{m=0}^{1} \frac{(-1)^{m}\left[\delta_{j_{1}}\left(t / \lambda_{j_{1}}\right)^{\beta_{j_{1}}}\right]^{m}}{m !} \sum_{m=0}^{l} \frac{(-1)^{m}\left[\delta_{j_{2}}\left(t / \lambda_{j_{2}}\right)^{\beta_{j_{2}}}\right]^{m}}{m !}\right. \\
& +\vartheta_{13} \sum_{m=0}^{1} \frac{(-1)^{m}\left[\delta_{j_{1}}\left(t / \lambda_{j_{1}}\right)^{\beta_{j_{1}}}\right]^{m}}{m !} \sum_{m=0}^{l} \frac{(-1)^{m}\left[\delta_{j_{3}}\left(t / \lambda_{j_{3}}\right)^{\beta_{j_{3}}}\right]^{m}}{m !} \\
& +\vartheta_{23} \sum_{m=0}^{1} \frac{(-1)^{m}\left[\delta_{j_{2}}\left(t / \lambda_{j_{2}}\right)^{\beta_{j_{2}}}\right]^{m}}{m !} \sum_{m=0}^{l} \frac{(-1)^{m}\left[\delta_{j_{3}}\left(t / \lambda_{j_{3}}\right)^{\beta_{3}}\right]^{m}}{m !} \\
& \left.+\vartheta_{123} \prod_{k=1}^{3} \sum_{m=0}^{l} \frac{\left.(-1)^{m}\left[\delta_{j_{k}}\left(t / \lambda_{j_{k}}\right)^{\beta_{j_{k}}}\right]^{m}\right]}{m !}\right] \prod_{k=1}^{3}\left[1-\sum_{m=0}^{l} \frac{(-1)^{m}\left[\delta_{j_{k}}\left(t / \lambda_{j_{k}}\right)^{\beta_{j_{k}}}\right]^{m}}{m !}\right] \\
& =\varphi(\rho)
\end{aligned}
$$

where:

$$
\left\{\begin{array}{ccc}
\delta_{j_{k}}=\rho_{j_{k}}, & l=3 & \text { if } j_{k} \in r \\
\delta_{j_{k}}=1, & l=\infty & \text { if } j_{k} \notin r,
\end{array} \text { for } k=1,2,3\right.
$$

The reliability equivalence factors can be obtained as the solutions of the nonlinear Equation 19. 


\section{Real Time Data Application}

In many cases the values of the parameters of Weibull distribution are unknown and need to be estimated from real data. Here, real time data from Murthy et al. (2004) are used to obtain the maximum likelihood estimators for parameters of Weibull distribution which can be computed from the following relations:

$\hat{\lambda}=\frac{1}{n} \sum_{i=1}^{n} t_{i} \hat{\beta}, \hat{\beta}=\left[\frac{\sum_{i=1}^{n} t_{i}^{\hat{\beta}} \ln \left(t_{i}\right)}{\sum_{i=1}^{n} t_{i}^{\hat{\beta}}}-\frac{1}{n} \sum_{i=1}^{n} \ln \left(t_{i}\right)\right]^{-1}$

Data set (1) complete data for failure times of 20 electric bulbs: $1.32,12.37,6.56,5.05,11.58,10.56$, $21.82,3.60,1.33,12.62,5.36,7.71,3.53,19.61,36.63$, $0.39,21.35,7.22,12.42$ and 8.92:

The maximum likelihood estimators of $\lambda$ and $\beta$ for the first unit can be obtained using relations (20) and the results are:

$$
\hat{\lambda}_{1}=18.604, \hat{\beta}_{1}=1.211
$$

Data set (2) complete data for lifetimes of 20 electronic components: $0.03,0.12,0.22,0.35,0.73,0.79$, $1.25,1.41,1.52,1.79,1.80,1.94,2.38,2.40,2.87,2.99$, $3.14,3.17,4.72$ and 5.09 .

The maximum likelihood estimators of $\lambda$ and $\beta$ for the second unit can be obtained using relations (20) and the results are:

$$
\hat{\lambda}_{2}=2.344, \hat{\beta}_{2}=1.196
$$

Data set (3) complete data for failure times of 20 components: $0.481,1.196,1.438,1.797,1.811,1.831$, $1.885,2.104,2.133,2.144,2.282,2.322,2.334,2.341$, $2.428,2.447,2.511,2.593,2.715$ and 3.218 .

The maximum likelihood estimators of $\lambda$ and $\beta$ for the thrid unit can be obtained using relations (20) and the results are:

$$
\hat{\lambda}_{3}=36.026, \hat{\beta}_{3}=4.304
$$

Now, the following data will be considered:

$$
\begin{aligned}
& \vartheta_{12}=0.6, \vartheta_{13}=0.5, \vartheta_{23}=0.7, \vartheta_{123}=1, \lambda_{s_{1}} \\
& =20, \lambda_{s_{2}}=5, s_{s_{3}}=40, \beta_{s_{1}}=\beta_{s_{2}}=\beta_{s_{3}}=1
\end{aligned}
$$

Comparison of the reliability function of the original system and the improved systems according to reduction method, hot duplication method, cold and warm duplication methods is obtained in Fig. 5.

In Fig. 5, it can be observed that the reliability function of the original system is improved by using four methods: (i) Reduction method, (ii) cold duplication method, (iii) warm duplication method and (iv) hot duplication method. Applying the reduction method is the better one. Applying the cold duplication method is better than applying the hot duplication method which is better than applying the warm duplication method.

In Fig. 6, comparison of the reliability functions of the improved system for different values of $\rho$ versus time is shown, where $\rho$ differs from 0 to 1 and $r=\{1,2,3\}$. It can be observed that decreasing the value of the equivalence factor $\rho$ increasing the value of the reliability function of the system.

In Fig. 7, reliability function of the improved system according cold standby method versus time is shown for different sets c. It can be observed that increasing the number of the units which duplicated by a cold standby unit increases the system reliability.

In Fig. 8, reliability function of the improved system according hot standby method versus time is shown for different sets $h$. It can be observed that increasing the number of the units which duplicated by a hot standby unit increases the system reliability.

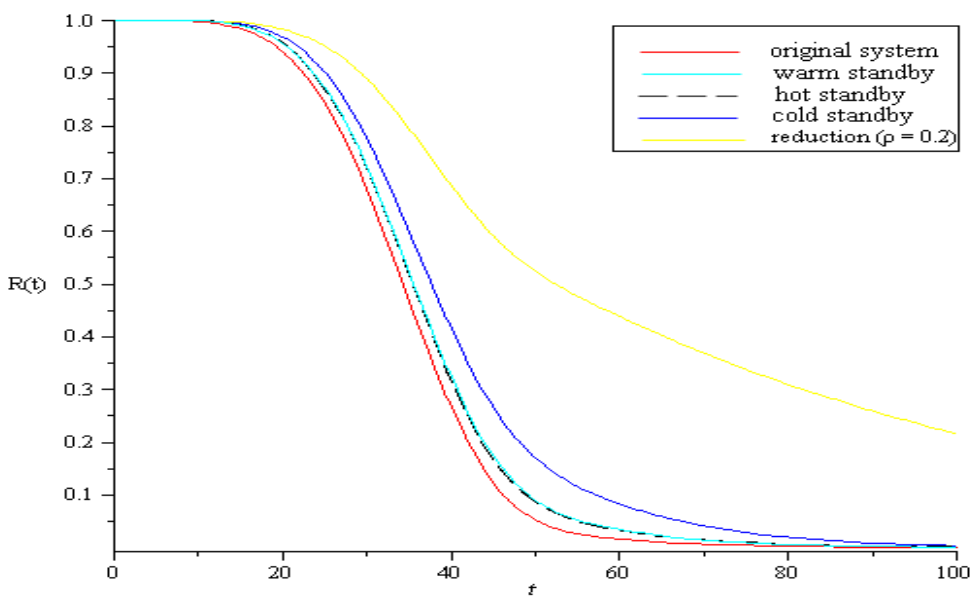

Fig. 5: Comparison of reliability functions of original system and improved systems 


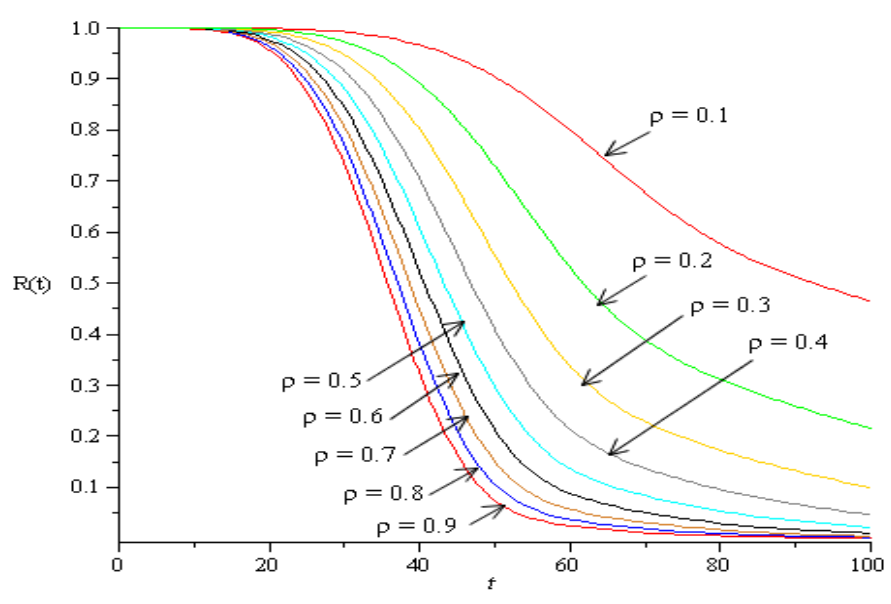

Fig. 6: Comparison of reliability functions of the improved system for different values of $\rho$ where $r=\{1,2,3\}$

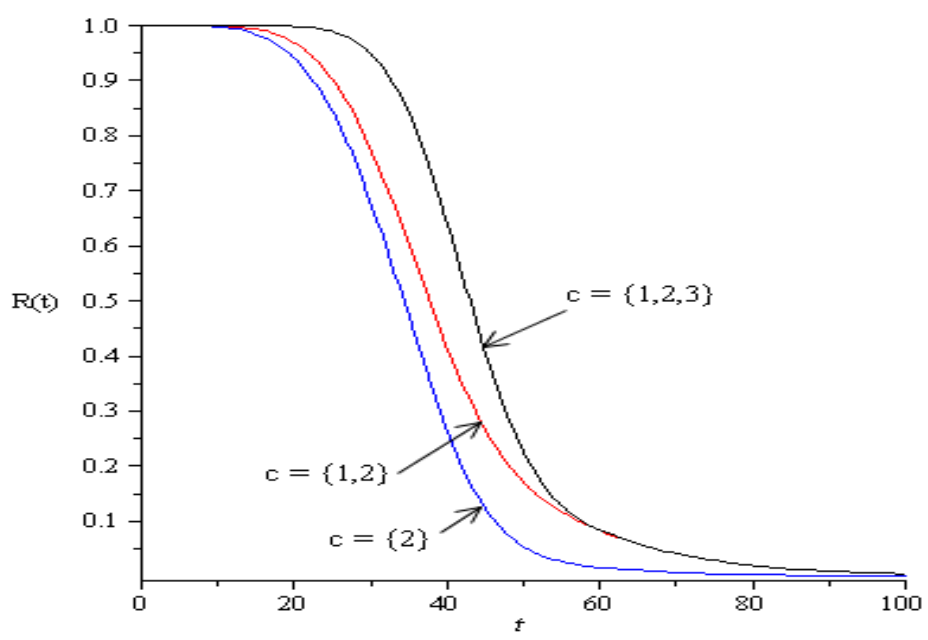

Fig. 7: Reliability function of the improved system according cold standby method

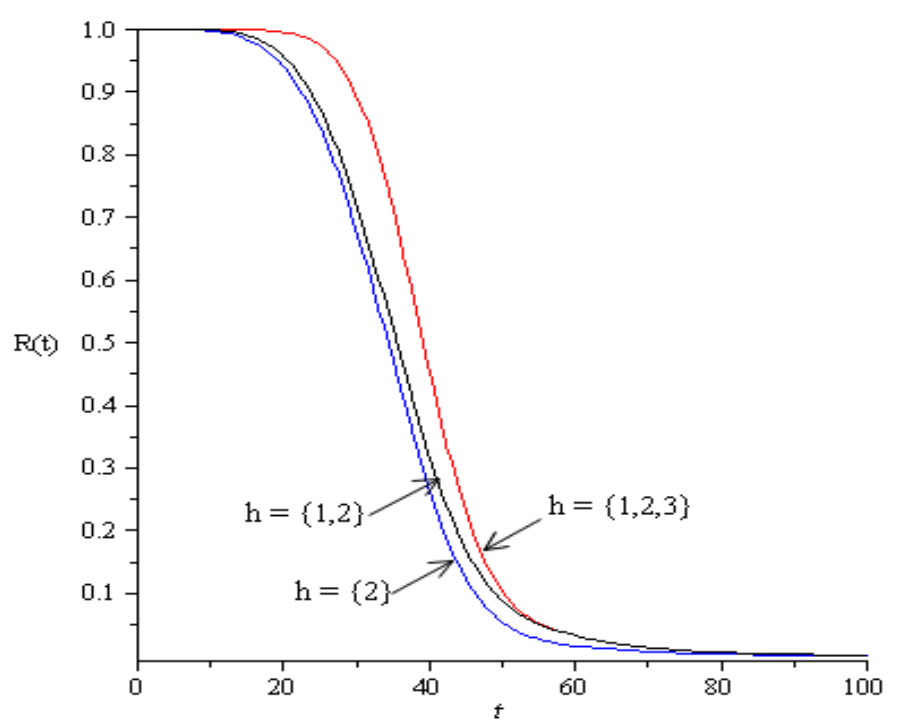

Fig. 8: Reliability function of the improved system according hot standby method 


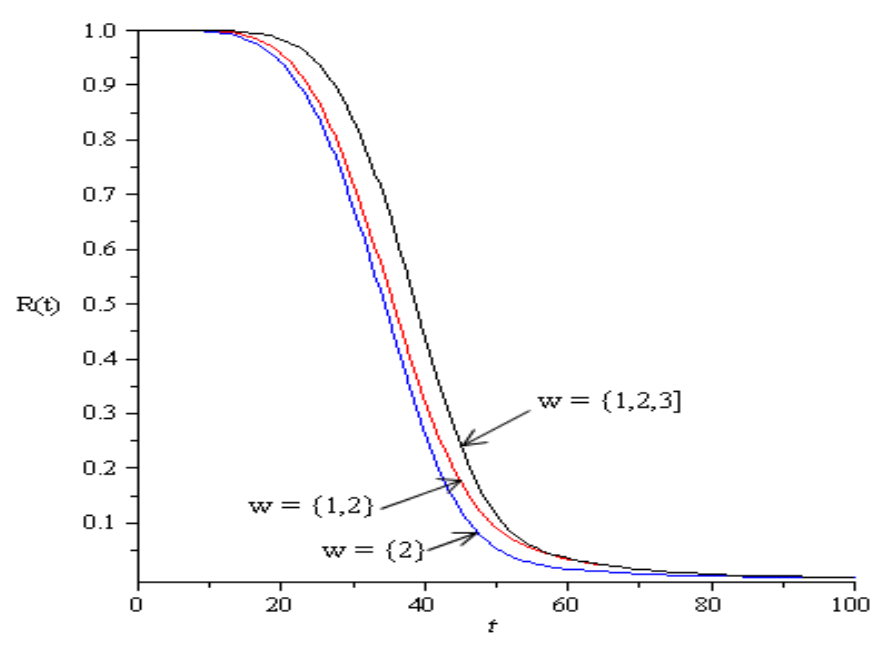

Fig. 9: Reliability function of the improved system according warm standby method

Table 1: Reliability values of the improved system according to cold duplication method at time $t=30$

\begin{tabular}{llllllll}
\hline$c$ (different sets) & $\{1\}$ & $\{2\}$ & $\{3\}$ & $\{1,2\}$ & $\{1,3\}$ & $\{2,3\}$ & $\{1,2,3\}$ \\
\hline$R(\mathrm{t})$ & 0.77661 & 0.67974 & 0.93112 & 0.77661 & 0.95027 & 0.93112 & 0.95027
\end{tabular}

Table 2: Reliability values of the improved system according to hot duplication method at time $t=30$

\begin{tabular}{|c|c|c|c|c|c|c|c|}
\hline$h$ (different sets) & $\{1\}$ & $\{2\}$ & $\{3\}$ & $\{1,2\}$ & $\{1,3\}$ & $\{2,3\}$ & $\{1,2,3\}$ \\
\hline$R(\mathrm{t})$ & 0.72234 & 0.67974 & 0.88080 & 0.72234 & 0.89523 & 0.88080 & 0.89523 \\
\hline$w$ (different sets) & $\{1\}$ & $\{2\}$ & $\{3\}$ & $\{1,2\}$ & $\{1,3\}$ & $\{2,3\}$ & $\{1,2,3\}$ \\
\hline$R(\mathrm{t})$ & 0.72364 & 0.67975 & 0.81607 & 0.72364 & 0.83972 & 0.81607 & 0.83972 \\
\hline
\end{tabular}

Table 4: Reliability equivalence factors $\rho_{\text {cold }}$ for different sets $r$ and $c$ at time $t=30$

\begin{tabular}{|c|c|c|c|c|c|c|c|}
\hline $\mathrm{r}$ & $\{1\}$ & $\{2\}$ & $\{3\}$ & $\{1,2\}$ & $\{1,3\}$ & $\{2,3\}$ & $\{1,2,3\}$ \\
\hline$\{1\}$ & 0.41335 & 0.73986 & 0.09003 & 0.41335 & 0.06282 & 0.09003 & 0.06282 \\
\hline$\{2\}$ & 0.03600 & 0.07565 & 0.00717 , & 0.03600 & 0.00498 & 0.00717 & 0.00498 , \\
\hline & 0.12114 & 0.10661 & 0.13130 & 0.12114 & 0.13222 & 0.13130 & 0.13222 \\
\hline$\{3\}$ & 0.63943 & 0.99449 & 0.17576 & 0.63943 & 0.12525 & 0.17576 & 0.12525 \\
\hline$\{1,2\}$ & -ve & -ve & -ve & -ve & $\begin{array}{l}0.06275 \\
0.10132\end{array}$ & -ve & $\begin{array}{r}0.06275 \\
0.10132\end{array}$ \\
\hline$\{1,3\}$ & 0.67989 & 0.86821 & 0.30595 & 0.67989 & 0.24947 & 0.30595 & 0.24947 \\
\hline$\{2,3\}$ & - ve & - ve & - ve & ve & - ve & - ve & - ve \\
\hline$\{1,2,3\}$ & $>1$ & $>1$ & $>1$ & $>1$ & $>1$ & $>1$ & $>1$ \\
\hline
\end{tabular}

Table 5: Reliability equivalence factors $\rho_{\text {hot }}$ for different sets $r$ and $h$ at time $t=30$

\begin{tabular}{|c|c|c|c|c|c|c|c|}
\hline$r$ & $\{1\}$ & $\{2\}$ & $\{3\}$ & $\{1,2\}$ & $\{1,3\}$ & $\{2,3\}$ & $\{1,2,3\}$ \\
\hline$\{1\}$ & 0.58919 & 0.73986 & 0.17194 & 0.58919 & 0.14671 & 0.17194 & 0.14671 \\
\hline \multirow[t]{2}{*}{2} & 0.05536 & 0.07565 , & 0.01392 & 0.05536 , & 0.01181 , & 0.01392 , & 0.01181 , \\
\hline & 0.11505 & 0.10661 & 0.12861 & 0.11505 & 0.12942 & 0.12861 & 0.12942 \\
\hline$\{3\}$ & 0.83116 & 0.99449 & 0.31513 & 0.83116 & 0.27413 & 0.31513 & 0.27413 \\
\hline$\{1,2\}$ & -ve & -ve & -ve & -ve & -ve & -ve & -ve \\
\hline$\{1,3\}$ & 0.78946 & 0.86821 & 0.43935 & 0.78946 & 0.40264 & 0.43935 & 0.40264 \\
\hline$\{2,3\}$ & -ve & -ve & -ve & -ve & -ve & -ve & -ve \\
\hline$\{1,2,3\}$ & $>1$ & $>1$ & $>1$ & $>1$ & $>1$ & $>1$ & $>1$ \\
\hline
\end{tabular}


Table 6: Reliability equivalence factors $\rho_{\text {warm }}$ different sets $r$ and $w$ at time $t=30$

\begin{tabular}{|c|c|c|c|c|c|c|c|}
\hline \multirow[b]{2}{*}{$r$} & \multicolumn{6}{|c|}{$w$} & \multirow[b]{2}{*}{$1,2,3$} \\
\hline & $\{1\}$ & $\{2\}$ & $\{3\}$ & $\{1,2\}$ & $\{1,3\}$ & $\{2,3\}$ & \\
\hline$\{1\}$ & 0.58467 & 0.73983 & 0.30766 & 0.58467 & 0.25324 & 0.30766 & 0.25324 \\
\hline$\{2\}$ & 0.05482 , & 0.07565 , & 0.02582 , & 0.05482 & 0.02092 & 0.02582 & 0.02092, \\
\hline & 0.11523 & 0.10662 & 0.12439 & 0.11523 & 0.12606 & 0.12439 & 0.12606 \\
\hline$\{3\}$ & 0.82636 & 0.99445 & 0.51036 & 0.82636 & 0.43678 & 0.51036 & 0.43678 \\
\hline$\{1,2\}$ & -ve & -ve & -ve & -ve & -ve & -ve & -ve \\
\hline$\{1,3\}$ & 0.78696 & 0.86819 & 0.59350 & 0.78696 & 0.53901 & 0.59350 & 0.53901 \\
\hline$\{2,3\}$ & -ve & -ve & -ve & -ve & -ve & $-\mathrm{ve}$ & -ve \\
\hline$\{1,2,3\}$ & $>1$ & $>1$ & $>1$ & $>1$ & $>1$ & $>1$ & $>1$ \\
\hline
\end{tabular}

In Fig. 9, reliability function of the improved system according warm standby method versus time is shown for different sets w. It can be observed that increasing the number of the units which duplicated by a warm standby unit increases the system reliability.

In Table 1, it can be observed that using cold duplication method for the unit $\{1\}$, for example, increases the reliability function of the system from 0.67974 to 0.77661 and applying this method for the units $\{1,2,3\}$ increases the reliability function of the system from 0.67974 to 0.95027 . So, increasing the number of the units which connected by a cold standby unit increases the system reliability.

In Table 2, it can be observed that using hot duplication method for the unit $\{1\}$, for example, increases the reliability function of the system from 0.67974 to 0.72234 and applying this method for the units $\{1,2,3\}$ increases the reliability function of the system from 0.67974 to 0.89523 . So, increasing the number of the units which duplicated by a hot standby unit increases the system reliability.

In Table 3, it can be observed that using warm standby method for the unit $\{1\}$, for example, increases the reliability function of the system from 0.67974 to 0.72364 and applying this method for the units $\{1,2,3\}$ increases the reliability function of the system from 0.67974 to 0.83972 . So, increasing the number of the units which connected by a warm standby unit increases the system reliability.

From the results obtained in Table 4, it can be observed that:

(1) The design with $R_{\text {cold }}(30)=0.93112$ where $\mathrm{c}=$ $\{2,3\}$ (for example) can be done by one of the following:

i. Reducing the failure rate of the unit $\{1\}$ by the factor $\rho_{\text {cold }}=0.09003$

ii. Reducing the failure rate of the unit $\{2\}$ by the factor $\rho_{\text {cold }}=0.00717$ or $\rho_{\text {cold }}=0.13130$

iii. Reducing the failure rate of the unit $\{3\}$ by the factor $\rho_{\text {cold }}=0.17576$.

iv. Reducing the failure rate of the units $\{1,3\}$ by the factor $\rho_{\text {cold }}=0.30595$
(2) All the values obtained for reliability equivalence factors when $r=\{2,3\}$ and some values when $r=\{1,2\}$ are negative and these is not acceptable since these factors must lie between 0 and 1. Also, for $r=\{1,2,3\}$ all the values obtained for reliability equivalence factors are greater than 1 and rejected.

From the results obtained in Table 5, it can be observed that:

(1) The design with $R_{h o t}(30)=0.88080$ where $\mathrm{h}=$ $\{2,3\}$ (for example) can be done by one of the following:

i. Reducing the failure rate of the unit $\{1\}$ by the factor $\rho_{\text {hot }}=0.17194$

ii. Reducing the failure rate of the unit $\{2\}$ by the factor $\rho_{\text {hot }}=0.01392$ or $\rho_{\text {hot }}=0.12861$

iii. Reducing the failure rate of the unit $\{3\}$ by the factor $\rho_{\text {hot }}=0.31513$

iv. Reducing the failure rate of the units $\{1,3\}$ by the factor $\rho_{\text {hot }}=0.43935$

(2) All the values obtained for reliability equivalence factors when $r=\{2,3\}$ and $r=\{1$, $2\}$ are negative and exceed 1 when $r=\{1,2,3\}$; hence all these values are rejected.

From the results obtained in Table 6 , it can be observed that:

(3) The design with $R_{\text {warm }}(30)=0.81607$ where c $=$ $\{2,3\}$ (for example) can be done by one of the following:

i. Reducing the failure rate of the unit $\{1\}$ by the factor $\rho_{\text {warm }}=0.30766$

ii. Reducing the failure rate of the unit $\{2\}$ by the factor $\rho_{\text {warm }}=0.02582$ or $\rho_{\text {warm }}=0.12439$

iii. Reducing the failure rate of the unit $\{3\}$ by the factor $\rho_{\text {warm }}=0.51036$

iv. Reducing the failure rate of the units $\{1,3\}$ by the factor $\rho_{\text {warm }}=0.59350$ 
In Table 5 and 6 , it can be observed that all the values obtained for reliability equivalence factors when $r=\{2$, $3\}$ and $r=\{1,2\}$ are negative and exceed 1 when $r=\{1$, $2,3\}$; hence all these values are rejected.

\section{Conclusion}

In literature, the units of a system are usually assumed to be independent. However, in many cases analysis of systems with dependent units is needed. A system with dependent units $\square$ means that the failure of any unit of the system affected by the failure of other units of the system. Copula is a useful tool to construct the reliability function of systems which consists of dependent units. In this paper, analysis of reliability of a parallel system with dependent and non-identical units was introduced using the formula of Farlie-GumbelMorgenstern (FGM) copula in $\mathrm{n}$ dimension. Reduction method was introduced in order to improve the system reliability by reducing the failure rate of some of the system components. Also, redundancy methods were used to improve system reliability. These methods include hot duplication method, warm standby duplication method and cold standby duplication method. Reliability equivalence factors were introduced to compare between different improvement methods.

Future works can deal with different types of copula such as Gumbel-Hougaard copula, Clayton copula, Frank copula and so on. Other distributions can be used to model lifetimes of systems devices such as lognormal, exponentiated exponential, gamma and so on. The study of this paper can be applied on systems with more complex configuration such as series-parallel system, parallel-series system, bridge and so on. Also, the number of redundant units in cold and warm standby duplication methods can be increased to get a higher reliability.

\section{Acknowledgement}

I would thank the reviwers for their comments which improved my paper.

\section{Ethics}

This article is original and contains unpublished material. The corresponding author confirms that all of the other authors have read and approved the manuscript and there are no ethical issues involved.

\section{References}

El-Damcese, M.A. and D.S. Ayoub, 2011. Reliability equivalence factors of a parallel system in twodimensional distribution. J. Reliability Stat. Stud., 4: 33-42.
El-Damcese, M.A. and K.A. Alltifi, 2013. Reliability equivalence factor of a parallel system subject to time varying failure rates. Eng. Math. Lett., 2: 42-55.

El-Damcese, M.A., 2009. Reliability equivalence factors of a series-parallel system in Weibull distribution. Int. J. Math. Forum, 4: 941-951.

Ezzati, G. and A. Rasouli, 2015. Evaluating system reliability using linear-exponential distribution function. Int. J. Adv. Stat. Probability, 3: 15-24.

Gauvreau, K. and M. Pagano, 1997. The analysis of correlated binary outcomes using multivariate logistic regression. Biometr. J., 39: 309-325.

Hai, A., Y. Hang and H. Fukai, 2016. Analysis and application of mechanical system reliability model based on copula function. Polish Maritime Res., 23: 187-191. DOI: 10.1515/pomr-2016-0064

Khan, A.H. and T.R. Jan, 2015. Reliability evaluation of engineering system using modified Weibull distribution. Res. J. Math. Stat. Sci., 3: 1-8.

Liu, Y. and X. Fan, 2016. Time-independent reliability analysis of bridge system based on mixed copula models. Math. Problems Eng.

Mangey, R. and S.B. Singh, 2009. Analysis of reliability characteristics of a complex engineering system under copula. J. Reliability Stat. Stud., 2: 91-102.

Migdadi, H.S. and M.S. Al-Batah, 2014. Testing reliability equivalence factors of a series-parallel systems in Burr type X distribution. Brit. J. Math. Comput. Sci., 4: 2618-2629.

Murthy, D.P., M. Xie and R. Jiang, 2004. Weibull Models. 1st Edn., Wiley-Interscience, Canada.

Mustafa, A. and A. El-Faheem, 2014. Reliability equivalence factors of a system with mixture of $n$ independent and non-identical lifetimes with delay time. J. Egyptian Math. Society, 22: 96-101.

Mustafa, A., 2009. Reliability equivalence factors for some systems with mixture weibull failure rates. African J. Math. Comput. Sci. Res., 2: 6-13.

Nelsen, R.B., 2006. An Introduction to Copulas. 1st Edn., Springer-Verlag, New York.

R॰de, L., 1989. Reliability equivalence: Studies in statistical quality control and reliability. Math. Stat.

R॰de, L., 1993a. Reliability equivalence. J. Microelectrons Reliability, 33: 323-325.

R॰de, L., 1993b. Reliability survival equivalence. J. Microelectrons Reliability, 33: 881-894.

Sarhan, A.M. and A. Mustafa, 2006. Reliability equivalence of a series system consists of $n$ independent and non-identical components. Int. J. Reliability Applic., 7: 111-125.

Sarhan, A.M., 2000. Reliability equivalence of independent and non-identical components series systems. J. Reliability Eng. Syst. Safety, 67: 293-300. 
Sarhan, A.M., 2002. Reliability equivalence with a basic series/parallel system. J. Applied Math. Comput., 132: $115-133$.

Sarhan, A.M., 2004. Reliability equivalence factors of a bridge network system. Int. J. Reliability Applic., 5: 81-103.

Sarhan, A.M., 2005. Reliability equivalence factors of parallel system. Reliability Eng. Syst. Safety, 87: 405-411.

Sarhan, A.M., 2009. Reliability equivalence factors of a general series-parallel system. J. Reliability Eng. Syst. Safety, 94: 229-236.

Sarhan, A.M., A.S. Al-Ruzaiza and I.A. El-Gohary, 2004. Reliability equivalence of a series-parallel system. J. Applied Math. Computat., 154: 257-277. DOI: 10.1016/S0096-3003(03)00709-4
Sarhan, A.M., L. Tadj, A. Al-khedhairi and A. Mustafa, 2008. Equivalence factors of a parallel-series system. J. Applied Sci., 10: 219-230.

Shawky, A.I., Y.H. Abdelkader and M.I. Al-Ohally, 2013. Reliability equivalence factors in exponentiated exponential distribution. Wulfenia J., 20: 75-85.

Xia, Y. and G. Zhang, 2007. Reliability equivalence factors in Gamma distribution. J. Applied Math. Computat., 187: 567-573. 